\title{
Entropy Numbers of General Diagonal Operators
}

\author{
Thomas KüHN \\ Mathematisches Institut \\ Universität Leipzig \\ Augustusplatz 10-11 \\ D-04109 Leipzig — Germany \\ kuehn@math.uni-leipzig.de
}

Recibido: 10 de febrero de 2005

Aceptado: 26 de abril de 2005

\begin{abstract}
We determine the asymptotic behavior of the entropy numbers of diagonal operators $D: \ell_{p} \rightarrow \ell_{q},\left(x_{k}\right) \mapsto\left(\sigma_{k} x_{k}\right), 0<p, q \leq \infty$, under mild regularity and decay conditions on the generating sequence $\left(\sigma_{k}\right)$. Our results extend the known estimates for polynomial and logarithmic diagonals $\left(\sigma_{k}\right)$. Moreover, we also consider some exotic intermediate examples like $\sigma_{k}=\exp (-\sqrt{\log k})$.
\end{abstract}

Key words: entropy numbers, diagonal operators, $\ell_{p}$-spaces.

2000 Mathematics Subject Classification: 47B06 , 46B45.

\section{Introduction}

During the last decade there has been a considerable interest in entropy numbers of Sobolev embeddings, see e.g. the monograph by Edmunds and Triebel [4], the article [6] (and the references given therein), or the very recent papers $[3,10-12]$. This interest has its origin in applications to spectral theory of (pseudo-)differential operators, via the famous Carl-Triebel inequality. For details we refer to [4].

Estimating entropy numbers of function space embeddings is not an easy task. The first step is usually a reduction to a simpler problem in sequence spaces, using wavelet bases, atomic or subatomic decompositions. The resulting sequence spaces,

Supported in part by Ministerio de Educación y Ciencia de España (Project No. MTM200401888). 
however, are still quite complicated, often they are of mixed-norm type and/or involve weights. Therefore a further reduction is necessary, which leads to diagonal operators in $\ell_{p}$-spaces. This connection is our main motivation in the present paper.

In most cases where optimal entropy estimates for diagonal operators are known, the corresponding diagonal is of a very special form, e.g., $\sigma_{k} \sim k^{-\alpha}$ or $\sigma_{k} \sim(\log k)^{-\alpha}$ (see $[1,3,9])$. We want to establish sharp upper and lower bounds under less restrictive assumptions. Our general results (Theorems 2.2, 3.2, and 3.3) apply to many concrete cases, e.g., to all sequences of polynomial type $(\mathrm{P})$ or logarithmic type (L), as defined in section 1. Moreover, we give almost sharp estimates for some interesting intermediates examples like $\sigma_{k}=\exp (-\sqrt{\log k})$.

The organization of the paper is as follows: In section 1 we fix notation and state some known results for later use, in sections 2 and 3 we consider polynomial respectively logarithmic diagonals, and in section 4 we deal with the intermediate case.

\section{Notation and preliminaries}

Now we fix notation and state some preliminary results which will be needed later. Let $X$ and $Y$ be quasi-Banach spaces. The $k$-th entropy number of a bounded linear operator $T: X \rightarrow Y$ is defined as

$$
e_{k}(T)=\inf \left\{\varepsilon>0: T\left(B_{X}\right) \text { can be covered by } 2^{k-1} \text { balls of radius } \varepsilon \text { in } Y\right\},
$$

where $B_{X}$ denotes the closed unit ball in $X$. Other related quantities are the approximation numbers

$$
a_{k}(T)=\inf \{\|T-L\|: \operatorname{rank} L<k\} .
$$

These numbers enjoy many nice properties like additivity, multiplicativity, etc., and entropy numbers behave, in addition, well under interpolation. The details (for operators in Banach spaces) can be found in the monographs $[7,13]$ (see also [4]), concerning the extension to quasi-Banach spaces. Clearly, an operator $T$ is compact if and only if $\lim _{k \rightarrow \infty} e_{k}(T)=0$, thus the rate of decay of the sequence $\left(e_{k}(T)\right)$ describes the 'degree' of compactness of $T$. In the sequel we need the following relation between approximation and entropy numbers.

For every $\alpha>0$ there is a constant $c_{\alpha}>0$ such that

$$
\sup _{k \leq n} k^{\alpha} e_{k}(T) \leq c_{\alpha} \cdot \sup _{k \leq n} k^{\alpha} a_{k}(T) \quad \text { for all } n \in \mathbb{N} .
$$

The properties of entropy numbers imply that

$$
L_{\alpha}^{(e)}(T):=\sup _{k \in \mathbb{N}} k^{\alpha} e_{k}(T)
$$

defines a quasi-norm (on the vector space of all operators $T: X \rightarrow Y$ for which this quantity is finite). In fact, this quasi-norm gives rise to a complete quasi-normed operator ideal in the sense of Pietsch [13]. 
As usual we denote by $\ell_{p}, 0<p<\infty$, the space of all (real or complex) sequences $x=\left(x_{k}\right)$ such that

$$
\|x\|_{p}:=\left(\sum_{k=1}^{\infty}\left|x_{k}\right|^{p}\right)^{1 / p}<\infty
$$

(with the usual modification for $p=\infty$ ). Then $\left(\ell_{p},\|\cdot\|_{p}\right)$ is a quasi-Banach space (even a Banach space for $1 \leq p \leq \infty$ ). By $\ell_{p}^{n}$ we denote $\mathbb{R}^{n}$ resp. $\mathbb{C}^{n}$ equipped with the analogue of $\|\cdot\|_{p}$.

The following basic result is essentially due to Schütt [14], who considered Banach spaces. The extension to the quasi-Banach case can be found in [4] (see also the supplement in [8]), which will be used later.

Let $0<p<q \leq \infty$, and set $\lambda:=1 / p-1 / q$. Then one has

$$
e_{k}\left(\text { id }: l_{p}^{n} \rightarrow l_{q}^{n}\right) \sim \begin{cases}1 & \text { if } 1 \leq k \leq \log n \\ \left(\frac{\log \left(1+\frac{n}{k}\right)}{k}\right)^{\lambda} & \text { if } \log n \leq k \leq n \\ 2^{-\frac{k-1}{n}} n^{-\lambda} & \text { if } k \geq n\end{cases}
$$

for real spaces. For complex spaces the same result holds, if we replace on the righthand side $n$ by $2 n$. As a consequence we obtain in both cases

$$
L_{\alpha}^{(e)}\left(\mathrm{id}: l_{p}^{n} \rightarrow l_{q}^{n}\right) \sim n^{\alpha-\lambda} \quad \text { whenever } \quad \alpha>\lambda .
$$

If $\sigma=\left(\sigma_{k}\right)$ is a scalar sequence, then we denote by $D_{\sigma}$ the mapping $\left(x_{k}\right) \mapsto\left(\sigma_{k} x_{k}\right)$. This defines a bounded linear operator $D_{\sigma}: \ell_{p} \rightarrow \ell_{q}$ if and only if $\sigma \in \ell_{r}$ with $\frac{1}{r}+\frac{1}{p} \geq \frac{1}{q}$. Since we are interested in compact operators, we can assume that $\lim _{k \rightarrow \infty} \sigma_{k}=0$, and by the properties of the (quasi-)norms in $\ell_{p}$ and $\ell_{q}$ we can furthermore assume that $\left(\sigma_{k}\right)$ is real and non-increasing.

Clearly we cannot expect to obtain the correct entropy behavior for arbitrary diagonal operators, without any condition on the generating diagonal. In fact, the entropy numbers may behave quite irregular. Therefore we have to pose certain reasonable regularity and decay conditions. On one hand they should be general enough, so that many natural examples are included, but on the other hand they should also be easy to check. In order to find out appropriate conditions, we list and analyze some relevant examples which should at least be covered.

Given two sequences of positive real numbers $\left(a_{k}\right)$ and $\left(b_{k}\right)$ we write $a_{k} \preceq b_{k}$, if there is a constant $c>0$ such that $a_{k} \leq c \cdot b_{k}$ for all $k \in \mathbb{N}$, while $a_{k} \sim b_{k}$ will stand for $a_{k} \preceq b_{k} \preceq a_{k}$. Logarithms are always taken in base $2, \log =\log _{2}$.

Examples. Let $\alpha>0, \beta \in \mathbb{R}$ and $0<\delta<1$.

(i) $\sigma_{k} \sim k^{-\alpha}$.

(ii) $\sigma_{k} \sim k^{-\alpha}(\log k)^{\beta}$. 
(iii) $\sigma_{k} \sim(\log k)^{-\alpha}$.

(iv) $\sigma_{k} \sim(\log k)^{-\alpha}(\log \log k)^{\beta}$.

(v) $\sigma_{k} \sim(\log \ldots \log k)^{-\alpha} \quad(m$-fold iterated logarithm).

(vi) $\sigma_{k} \sim \exp \left(-\alpha(\log k)^{\delta}\right)$.

First we observe that all examples satisfy the doubling condition

$$
\sigma_{k} \sim \sigma_{2 k}
$$

For non-increasing sequences one easily verifies that this is equivalent to

$$
\inf _{n \geq k} \frac{\sigma_{n}}{\sigma_{k}} \cdot\left(\frac{n}{k}\right)^{a}>0 \quad \text { for some } a>0 .
$$

Similar conditions play an important role in [2]. In particular this implies $\sigma_{k} \succeq k^{-a}$, i.e., $\left(\sigma_{k}\right)$ cannot decay faster than polynomially.

For examples (i) and (ii) one has, in addition,

$$
\sup _{n \geq k} \frac{\sigma_{n}}{\sigma_{k}} \cdot\left(\frac{n}{k}\right)^{b}<\infty \quad \text { for some } b>0,
$$

implying in particular the decay condition $\sigma_{k} \preceq k^{-b}$.

In examples (iii)-(v) even the dyadic subsequences satisfy the doubling condition (3), we call this the dyadic doubling condition. For non-increasing sequences it is equivalent to

$$
\sigma_{k} \sim \sigma_{k^{2}}
$$

and also to

$$
\inf _{n \geq k} \frac{\sigma_{n}}{\sigma_{k}} \cdot\left(\frac{1+\log n}{1+\log k}\right)^{a}>0 \quad \text { for some } a>0 .
$$

Moreover, we will use the similar condition

$$
\sup _{n \geq k} \frac{\sigma_{n}}{\sigma_{k}} \cdot\left(\frac{1+\log n}{1+\log k}\right)^{b}<\infty \quad \text { for some } b>0 .
$$

This brief analysis of the given examples shows that conditions (3)-(8) fit to our aims: They are sufficiently general and easy to check. Therefore we shall work with these conditions.

In order to extend and unify the list of examples, we define inductively the iterated logarithms (for $t \geq 1$ ) by

$$
L_{1}(t):=1+\log t \quad \text { and } \quad L_{j+1}(t):=1+\log L_{j}(t),
$$

and consider the following sequences of polynomial resp. logarithmic type. 


\section{More examples.}

$$
\begin{array}{rlrl}
\sigma_{k} & \sim k^{-\alpha} \prod_{j=1}^{m} L_{j}(k)^{a_{j}} & & \left(m \in \mathbb{N}, \alpha>0, a_{j} \in \mathbb{R}\right) \\
\sigma_{k} \sim \prod_{j=\ell}^{m} L_{j}(k)^{a_{j}} & & \left(1 \leq \ell \leq m, a_{\ell}<0, a_{\ell+1}, \ldots, a_{m} \in \mathbb{R}\right) .
\end{array}
$$

All examples in (P) satisfy (4) and the polynomial decay condition

$$
k^{-a} \preceq \sigma_{k} \preceq k^{-b} \quad \text { for some } \quad 0<b \leq a<\infty,
$$

while the examples in (L) satisfy (7) and the logarithmic decay condition

$$
\sigma_{k} \succeq(\log k)^{-a} \quad \text { for some } \quad 0<a<\infty \text {. }
$$

Obviously, all sequences in (i)-(v) are either of type $(\mathrm{P})$ or of type $(\mathrm{L})$. However, the examples under (vi) are quite interesting exceptions, they are of neither of these two types. Roughly speaking, they are between the logarithmic and the polynomial scales, more precisely one has

$$
k^{-\varepsilon} \preceq \sigma_{k} \preceq(\log k)^{-a} \quad \text { for all } \quad \varepsilon>0 \text { and all } a<\infty .
$$

\section{Polynomial diagonals}

In this section we investigate diagonal operators $D_{\sigma}$ generated by sequences which are between two powers, meaning that

$$
k^{-a} \preceq \sigma_{k} \preceq k^{-b} \quad \text { for some } \quad 0<b \leq a<\infty .
$$

Our starting point is the following result by Carl [1]. Using operator ideal techniques, he proved it in the context of Banach spaces, i.e., when $p, q \geq 1$. The proof, however, extends literally to the quasi-Banach case.

Proposition 2.1. Let $0<p, q \leq \infty$ and $\alpha>\max (1 / q-1 / p, 0)$. Then

$$
\sigma_{k} \sim k^{-\alpha} \quad \text { implies } \quad e_{k}\left(D_{\sigma}: \ell_{p} \rightarrow \ell_{q}\right) \sim k^{1 / q-1 / p-\alpha} .
$$

Note that the condition on $\alpha$ is necessary, provided we consider only power-like diagonals $\sigma_{k} \sim k^{-\alpha}$ with $\alpha>0$. Indeed, then the operator $D_{\sigma}$ acts boundedly from $\ell_{p}$ to $\ell_{q}$ if and only if $\alpha+1 / p>1 / q$.

We extend this result to more general sequences, including in particular all examples of polynomial type $(\mathrm{P})$. The special case $\sigma_{k} \sim k^{-\alpha}(\log k)^{\beta}$ was already shown (in the Banach space setting) in [9]. 
Theorem 2.2. Let $0<p, q \leq \infty$, and let $\sigma=\left(\sigma_{k}\right)$ be a non-increasing sequence satisfying the doubling condition $\sigma_{k} \sim \sigma_{2 k}$ and, in addition,

$$
\sup _{n \geq k} \frac{\sigma_{n}}{\sigma_{k}} \cdot\left(\frac{n}{k}\right)^{\alpha}<\infty \quad \text { for some } \alpha>\max (1 / q-1 / p, 0) \text {. }
$$

Then

$$
e_{k}\left(D_{\sigma}: \ell_{p} \rightarrow \ell_{q}\right) \sim k^{1 / q-1 / p} \cdot \sigma_{k} .
$$

Proof. For the upper estimate we use a standard factorization technique. Consider the diagonal operators

$$
D_{\tau}: \ell_{p} \rightarrow \ell_{q} \quad \text { with } \quad \tau_{k}=k^{-\alpha}
$$

and

$$
D_{\rho}: \ell_{q} \rightarrow \ell_{q} \quad \text { with } \quad \rho_{k}=k^{\alpha} \sigma_{k},
$$

leading to the factorization $D_{\sigma}=D_{\rho} D_{\tau}$. Let $P$ denote the projection in $\ell_{q}$ onto the first $k-1$ coordinates. By (10) we get for all $k \in \mathbb{N}$

$$
a_{k}\left(D_{\rho}\right) \leq\left\|D_{\rho}-D_{\rho} P\right\|=\sup _{n \geq k} \rho_{n} \preceq \rho_{k} .
$$

Our assumption $\sigma_{k} \sim \sigma_{2 k}$ implies that also the sequence $\left(\rho_{k}\right)$ satisfies the doubling condition, or, equivalently condition (4). From (1) we deduce

$$
e_{k}\left(D_{\rho}\right) \preceq a_{k}\left(D_{\rho}\right) \preceq \rho_{k} .
$$

(Alternatively we could work here with the results of Gordon, König, and Schütt [5], or more precisely with the extension of their results to the quasi-Banach case.) Taking into account Proposition 2.1 and the multiplicativity of entropy numbers we arrive at

$$
e_{2 k}\left(D_{\sigma}\right) \leq e_{k}\left(D_{\tau}\right) \cdot e_{k}\left(D_{\rho}\right) \preceq k^{1 / q-1 / p-\alpha} \cdot k^{\alpha} \sigma_{k}=k^{1 / q-1 / p} \cdot \sigma_{k},
$$

which is equivalent to the desired upper estimate.

The lower estimate follows immediately from (2), indeed

$$
e_{k}\left(D_{\sigma}: \ell_{p} \rightarrow \ell_{q}\right) \geq \sigma_{k} \cdot e_{k}\left(\mathrm{id}: \ell_{p}^{k} \rightarrow \ell_{q}^{k}\right) \sim k^{1 / q-1 / p} \cdot \sigma_{k}
$$

Remark 2.3. In fact the assumptions of the preceding theorem are quite general. This is illustrated by the following observation: Let $a, b$ be given real numbers with $\alpha<b<a<\infty$. Then there exists a sequence $\left(\sigma_{k}\right)$ satisfying the assumptions of the theorem, and such that $\sigma_{k_{n}} \sim k_{n}^{-a}$ and $\sigma_{j_{n}} \sim j_{n}^{-b}$ for certain subsequences $k_{n} \rightarrow \infty$ and $j_{n} \rightarrow \infty$. For the construction of such a sequence see, e.g., [11]. 


\section{Logarithmic diagonals}

Next we consider logarithmic diagonals. Our main results rely essentially on the following proposition, which was shown for $q=\infty$ in [3].

Proposition 3.1. Let $0<p<q \leq \infty$ and set $\lambda:=1 / p-1 / q$. Then

$$
\sigma_{k} \sim(\log k)^{-\lambda} \quad \text { implies } \quad e_{k}\left(D_{\sigma}: \ell_{p} \rightarrow \ell_{q}\right) \sim k^{-\lambda} .
$$

Proof. For the upper estimates we prefer to work with identities in weighted spaces. If $\left(w_{k}\right)$ is any sequence of positive real numbers, then the space $l_{q}\left(w_{k}\right)$ consists of all sequences $x=\left(x_{k}\right)$ such that $w x:=\left(w_{k} x_{k}\right) \in l_{q}$, and the quasi-norm is defined as $\left\|x\left|l_{q}\left(w_{k}\right)\|:=\| w x\right| l_{q}\right\|$. The properties of entropy numbers imply that

$$
e_{k}\left(D_{\sigma}: \ell_{p} \rightarrow \ell_{q}\right)=e_{k}\left(\mathrm{id}: \ell_{p} \rightarrow \ell_{q}\left(\sigma_{k}\right)\right)
$$

By [3, Theorem 3.3] one has for $0<p<\infty$

$$
e_{k}\left(\mathrm{id}: \ell_{p} \rightarrow \ell_{\infty}\left((1+\log k)^{-1 / p}\right)\right) \preceq k^{-1 / p},
$$

which covers the case $q=\infty$ in our proposition.

Now let $0<p<q<\infty$ and $\sigma_{k} \sim(1+\log k)^{-\lambda}$. Set $\tau_{k}:=(1+\log k)^{-1 / p}$, and select $0<\theta<1$ such that

$$
\frac{1}{q}=\frac{1-\theta}{p}+\frac{\theta}{\infty}=\frac{1-\theta}{p}
$$

This choice gives

$$
\theta / p=1 / p-1 / q=\lambda \quad \text { and } \quad \sigma_{k} \sim \tau_{k}^{\theta} .
$$

Then it is trivial to check that for all $\left(x_{k}\right) \in \ell_{p} \cap \ell_{\infty}\left(\tau_{k}\right)$ the estimate

$$
\left\|\left(\sigma_{k} x_{k}\right)\right\|_{q} \sim\left\|\left(\tau_{k}^{\theta} x_{k}\right)\right\|_{q} \leq\left\|\left(x_{k}\right)\right\|_{p}^{1-\theta} \cdot\left\|\left(\tau_{k} x_{k}\right)\right\|_{\infty}^{\theta}
$$

holds. In other words, the space $\ell_{q}\left(\sigma_{k}\right)$ is an intermediate space of $J$-type $\theta$ with respect to the couple $\left(\ell_{p}, \ell_{\infty}\left(\tau_{k}\right)\right)$, whence (11) and the interpolation property of the entropy numbers imply, with some constant $c>0$ independent of $k$,

$$
\begin{aligned}
e_{k}\left(D_{\sigma}: \ell_{p} \rightarrow \ell_{q}\right) & =e_{k}\left(\mathrm{id}: \ell_{p} \rightarrow \ell_{q}\left(\sigma_{k}\right)\right) \\
& \leq c\left\|\mathrm{id}: \ell_{p} \rightarrow \ell_{p}\right\|^{1-\theta} \cdot e_{k}\left(\mathrm{id}: \ell_{p} \rightarrow \ell_{\infty}\left(\tau_{k}\right)\right)^{\theta} \\
& \preceq k^{-\theta / p}=k^{-\lambda} .
\end{aligned}
$$

This is the desired upper bound.

The estimate from below is again a simple consequence of (2) and the properties of entropy numbers. Indeed, if $P$ now denotes the projection onto the first $2^{k}$ coordinates, then one has

$$
e_{k}\left(D_{\sigma}: \ell_{p} \rightarrow \ell_{q}\right) \geq e_{k}\left(D_{\sigma} P\right) \geq \min _{j \leq 2^{k}} \sigma_{j} \cdot e_{k}\left(\mathrm{id}: \ell_{p}^{2^{k}} \rightarrow \ell_{q}^{2^{k}}\right) \sim k^{-\lambda} .
$$


Now we are prepared to prove the main results of this section. It is an interesting phenomenon, observed already earlier in similar situations, that the entropy behavior of the logarithmic diagonal operators changes drastically, if the decay of the diagonal sequence is 'very slow'. So we have to distinguish two cases which will be treated separately. First we consider the case of 'fast logarithmic decay', meaning that $\sigma_{k} \preceq(\log k)^{-\lambda}$.

Theorem 3.2. Let $0<p<q \leq \infty$ and set $\lambda:=1 / p-1 / q$.

If $\sigma=\left(\sigma_{k}\right)$ is a non-increasing sequence such that

$$
\sigma_{k} \sim \sigma_{k^{2}} \quad \text { and } \quad \sup _{n \geq k} \frac{\sigma_{n}}{\sigma_{k}} \cdot\left(\frac{1+\log n}{1+\log k}\right)^{\lambda}<\infty,
$$

then

$$
e_{k}\left(D_{\sigma}: \ell_{p} \rightarrow \ell_{q}\right) \sim\left(\frac{\log k}{k}\right)^{\lambda} \sigma_{k}
$$

Proof. For the upper estimate we use the same method as in the proof of Theorem 2.2. Again we consider diagonal operators $D_{\tau}: \ell_{p} \rightarrow \ell_{q}$ and $D_{\rho}: \ell_{q} \rightarrow \ell_{q}$, but now with $\tau_{k}=(1+\log k)^{-\lambda}$ and $\rho_{k}=(1+\log k)^{\lambda} \sigma_{k}$, which gives the factorization $D_{\sigma}=D_{\rho} D_{\tau}$. By Proposition 3.1 we have

$$
e_{k}\left(D_{\tau}\right) \preceq k^{-\lambda} .
$$

Since the dyadic doubling condition implies the doubling condition (and only this is needed here), we can argue similarly as in the proof of Theorem 2.2 and we get

$$
e_{k}\left(D_{\rho}\right) \preceq \rho_{k}=(1+\log k)^{\lambda} \sigma_{k} .
$$

Thus the multiplicativity of entropy numbers yields

$$
e_{2 k}\left(D_{\sigma}\right) \leq e_{k}\left(D_{\rho}\right) \cdot e_{k}\left(D_{\tau}\right) \preceq \rho_{k} \cdot k^{-\lambda} \sim\left(\frac{\log k}{k}\right)^{\lambda} \sigma_{k} .
$$

Now we pass to the lower estimate. Fix $k \in \mathbb{N}$ and let $P$ be the projection onto the first $k^{2}$ coordinates. Then, using the dyadic doubling condition $\sigma_{k^{2}} \sim \sigma_{k}$ and the result in [8], we obtain the desired lower bound

$$
e_{k}\left(D_{\sigma}\right) \geq e_{k}\left(D_{\sigma} P\right) \geq \sigma_{k^{2}} \cdot e_{k}\left(\mathrm{id}: \ell_{p}^{k^{2}} \rightarrow \ell_{q}^{k^{2}}\right) \sim\left(\frac{\log k}{k}\right)^{\lambda} \sigma_{k}
$$

For 'very slow decay', i.e., if $\sigma_{k}$ decreases not faster than $(1+\log k)^{-\lambda}$, we have the following result.

Theorem 3.3. Let $0<p<q \leq \infty$ and set $\lambda:=1 / p-1 / q$.

If $\sigma=\left(\sigma_{k}\right)$ is a non-increasing sequence such that

$$
\inf _{n \geq k} \frac{\sigma_{n}}{\sigma_{k}} \cdot\left(\frac{1+\log n}{1+\log k}\right)^{\lambda}>0
$$


then

$$
e_{k}\left(D_{\sigma}: \ell_{p} \rightarrow \ell_{q}\right) \sim \sigma_{2^{k}}
$$

Proof. Fix $k \in \mathbb{N}$, let $P$ be the projection onto the first $2^{k}$ coordinates, and let $Q$ be the projection onto the remaining ones.

The lower estimate follows immediately from (2),

$$
e_{k}\left(D_{\sigma}\right) \geq e_{k}\left(D_{\sigma} P\right) \geq \sigma_{2^{k}} \cdot e_{k}\left(\mathrm{id}: \ell_{p}^{2^{k}} \rightarrow \ell_{q}^{2^{k}}\right) \sim \sigma_{2^{k}} .
$$

Now we prove the upper estimate. Since $\left(\sigma_{k}\right)$ is non-increasing, we have

$$
\left\|D_{\sigma} Q: \ell_{p} \rightarrow \ell_{q}\right\|=\sup _{j>2^{k}} \sigma_{j} \leq \sigma_{2^{k}} .
$$

In order to bound $e_{k}\left(D_{\sigma} P: \ell_{p} \rightarrow \ell_{q}\right)$ we use an appropriate factorization. Let $\tau_{j}=(1+\log j)^{-\lambda}$ for all $j \in \mathbb{N}$, and $\rho_{j}=(1+\log j)^{\lambda} \sigma_{j}$, for $j=1, \ldots, 2^{k}$ and $\rho_{j}=0$ for $j>2^{k}$. Proposition 3.1 and assumption (12) imply now that

$$
e_{k}\left(D_{\tau}: \ell_{p} \rightarrow \ell_{q}\right) \sim k^{-\lambda}
$$

and

$$
\left\|D_{\rho}: \ell_{q} \rightarrow \ell_{q}\right\|=\max _{j \leq 2^{k}} \rho_{j} \preceq \rho_{2^{k}} \sim k^{\lambda} \sigma_{2^{k}},
$$

whence the obvious factorization $D_{\sigma} P=D_{\rho} D_{\tau}$ gives

$$
e_{k}\left(D_{\sigma} P\right) \leq e_{k}\left(D_{\tau}\right)\left\|D_{\rho}\right\| \preceq k^{-\lambda} \cdot k^{\lambda} \sigma_{2^{k}}=\sigma_{2^{k}} .
$$

Thanks to (13), (14), and the additivity of entropy numbers we finally arrive at

$$
e_{k}\left(D_{\sigma}\right) \leq c \cdot\left(e_{k}\left(D_{\sigma} P\right)+\left\|D_{\sigma} Q\right\|\right) \preceq \sigma_{2^{k}},
$$

and the proof is finished.

Remark 3.4. Let us come back to the phenomenon mentioned above. Under the assumptions of Theorem 3.2 (roughly speaking if $\sigma_{k} \preceq(\log k)^{-\lambda}$ ) the entropy numbers of $D_{\sigma}: \ell_{p} \rightarrow \ell_{q}$ depend explicitly on $p$ and $q$. However, under the assumptions of Theorem 3.3 (roughly speaking for null sequences with $\sigma_{k} \succeq(\log k)^{-\lambda}$ ) the entropy numbers are independent of $p$ and $q$. Of course, an implicit dependence is still hidden in the critical exponent $\lambda=1 / p-1 / q$.

Remark 3.5. Theorems 3.2 and 3.3 yield, in particular, asymptotically sharp results for all diagonals of logarithmic type (L). In order to give a specific example which illustrates the phenomenon described in the previous remark, we state the following corollary. In the special case $\beta=0$ and for Banach spaces, i.e., for $1 \leq p<q \leq \infty$, this can be found in [9]. The completely different proof in [9] was based on probabilistic arguments, and used tools which are no longer available in the context of quasi-Banach spaces, e.g., complex interpolation, and Tomczak-Jaegermann's result [15] on duality of entropy numbers. 
Corollary 3.6. Let $0<p<q \leq \infty, \alpha>0$, and $\beta \in \mathbb{R}$. If $\lambda:=1 / p-1 / q$ and

$$
\sigma_{k} \sim(\log k)^{-\alpha}(\log \log k)^{\beta},
$$

then the entropy numbers $e_{k}:=e_{k}\left(D_{\sigma}: \ell_{p} \rightarrow \ell_{q}\right)$ behave asymptotically like

$$
e_{k} \sim\left\{\begin{array}{lll}
k^{-\lambda}(\log k)^{\lambda-\alpha}(\log \log k)^{\beta} & \text { if } \alpha>\lambda & \text { or } \quad \alpha=\lambda, \beta \leq 0 \\
k^{-\alpha}(\log k)^{\beta} & \text { if } \alpha<\lambda & \text { or } \quad \alpha=\lambda, \beta \geq 0 .
\end{array}\right.
$$

\section{The intermediate case}

In order to complement the results obtained so far, we finally study some diagonal operators $D_{\sigma}: \ell_{p} \rightarrow \ell_{q}$ with generating sequences between the logarithmic and the polynomial scales, in the sense of (9). Let us call this the intermediate case, typical sequences of this type are given in example (vi). The main aim in this section is to establish (almost) sharp entropy estimates for this example.

Before doing so, we state a more general result which is a direct consequence of the proofs of the lower estimate in Theorem 2.2 and the upper estimate in Theorem 3.2.

Proposition 4.1. Let $0<p<q \leq \infty$ and set $\lambda:=1 / p-1 / q$. If $\left(\sigma_{k}\right)$ is a nonincreasing sequence such that

$$
\sigma_{k} \sim \sigma_{2 k} \quad \text { and } \quad \sup _{n \geq k} \frac{\sigma_{n}}{\sigma_{k}} \cdot\left(\frac{1+\log n}{1+\log k}\right)^{\lambda}<\infty .
$$

Then

$$
k^{-\lambda} \sigma_{k} \preceq e_{k}\left(D_{\sigma}: \ell_{p} \rightarrow \ell_{q}\right) \preceq\left(\frac{\log k}{k}\right)^{\lambda} \sigma_{k} .
$$

Remark 4.2. In contrast to Theorem 3.2 we require here only the doubling condition $\sigma_{k} \sim \sigma_{2 k}$ instead of the dyadic doubling condition $\sigma_{k} \sim \sigma_{k^{2}}$, which is of course much stronger. Note that (15) implies

$$
k^{-\alpha} \preceq \sigma_{k} \preceq(\log k)^{-\lambda} \quad \text { for some } \quad \alpha>0 .
$$

Although lower and upper bounds in (16) are different, the result is optimal under assumption (15). For instance, by Propositions 2.1 and 3.1 we know:

- The lower bound is sharp if $\sigma_{k} \sim k^{-\alpha}$ for some $\alpha>0$.

- The upper bound is sharp if $\sigma_{k} \sim(\log k)^{-\lambda}$.

Our last theorem gives an almost optimal result for example (vi), indeed the gap between lower and upper bounds is very small. The result also shows that the asymptotic order of the entropy numbers $e_{k}\left(D_{\sigma}: \ell_{p} \rightarrow \ell_{q}\right)$ does not necessarily coincide with the upper or with the lower bound in (16). 
Theorem 4.3. Let $0<p<q \leq \infty, \alpha>0,0<\delta<1$, and

$$
\sigma_{k} \sim \exp \left(-\alpha(\log k)^{\delta}\right)
$$

Then one has, with $\lambda:=1 / p-1 / q$,

$$
\left(\frac{(\log k)^{1-\delta}}{k}\right)^{\lambda} \sigma_{k} \preceq e_{k}\left(D_{\sigma}: \ell_{p} \rightarrow \ell_{q}\right) \preceq\left(\frac{(\log k)^{1-\delta} \log \log k}{k}\right)^{\lambda} \sigma_{k} .
$$

Proof. Lower estimate. Fix $N \in \mathbb{N}$ and set $M:=N+\left[N^{1-\delta}\right]$, where $[x]$ denotes the greatest integer part of a real number $x$. This implies

$$
M^{\delta}-N^{\delta} \sim 1 \quad \text { and } \quad \sigma_{2^{N}} / \sigma_{2^{M}}=\exp \left(\alpha\left(M^{\delta}-N^{\delta}\right)\right) \sim 1
$$

Now the properties of entropy numbers and (2) yield

$$
\begin{aligned}
e_{2^{N}}\left(D_{\sigma}: \ell_{p} \rightarrow \ell_{q}\right) & \geq \sigma_{2^{M}} \cdot e_{2^{N}}\left(\mathrm{id}: \ell_{p}^{2^{M}} \rightarrow \ell_{q}^{2^{M}}\right) \\
& \sim \sigma_{2^{N}} \cdot 2^{-\lambda N}(M-N)^{\lambda} \sim \sigma_{2^{N}} \cdot 2^{-\lambda N} N^{(1-\delta) \lambda} .
\end{aligned}
$$

This proves the lower estimate for integers of the form $k=2^{N}$, and by a monotonicity argument it holds for all $k \in \mathbb{N}$.

Upper estimate. Given any natural number $N \in \mathbb{N}$, we now set

$$
M:=N+\left[C N^{1-\delta} \log N\right], \quad \text { where } \quad C=\frac{2^{1-\delta} \lambda}{\alpha \delta} .
$$

(Later in the proof we need that the constant $C$ is sufficiently large, and our specific choice will work.) Moreover, we use the following projections in $\ell_{p}$.

$$
\begin{array}{lll}
P: \quad \text { onto the coordinates } j \text { with } & 1 \leq j<2^{N}, \\
Q: & \text { onto the coordinates } j \text { with } & 2^{N} \leq j \leq 2^{M}, \\
R: \quad \text { onto the coordinates } j \text { with } & j>2^{M} .
\end{array}
$$

By the additivity of entropy numbers there is a constant $c=c(p, q)>0$ such that for all $k \in \mathbb{N}$ the inequality

$$
e_{3 k}\left(D_{\sigma}\right) \leq c \cdot\left(e_{k}\left(D_{\sigma} P\right)+e_{k}\left(D_{\sigma} Q\right)+e_{k}\left(D_{\sigma} R\right)\right)
$$

holds. We estimate the three summands separately, taking $k=2^{N}$.

First summand. Here we use operator ideal techniques. To this end we consider for $n=0, \ldots, N-1$ the projections $P_{n}$ onto the coordinates $j=2^{n}, \ldots, 2^{n+1}-1$ and split $D_{\sigma} P$ as

$$
D_{\sigma} P=\sum_{n=0}^{N-1} D_{\sigma} P_{n}
$$


Select now a real number $\rho>\lambda+1$. Moreover, note that there is a constant $c=$ $c(\alpha, \delta)>0$ such that

$$
\sigma_{k} \leq c \sigma_{m} \cdot \frac{m}{k} \quad \text { for all } \quad k \leq m,
$$

For the entropy ideal norms of $D_{\sigma} P_{n}$ this implies (with $k=2^{n}, m=2^{N}$ ),

$$
\begin{aligned}
L_{\rho}^{(e)}\left(D_{\sigma} P_{n}\right) & \leq \sigma_{2^{n}} \cdot L_{\rho}^{(e)}\left(\mathrm{id}: \ell_{p}^{2^{n}} \rightarrow \ell_{q}^{2^{n}}\right) \\
& \preceq 2^{N-n} \sigma_{2^{N}} \cdot 2^{(\rho-\lambda) n}=\sigma_{2^{N}} \cdot 2^{N+(\rho-\lambda-1) n} .
\end{aligned}
$$

From this estimate and the fact that the quasi-norm $L_{\rho}^{(e)}$ is equivalent to an $r$-norm for some $0<r<1$, we conclude

$$
\begin{aligned}
2^{\rho N} \cdot e_{2^{N}}\left(D_{\sigma} P\right) & \leq L_{\rho}^{(e)}\left(\sum_{n=0}^{N-1} D_{\sigma} P_{n}\right) \preceq\left(\sum_{n=0}^{N-1} L_{\rho}^{(e)}\left(D_{\sigma} P_{n}\right)^{r}\right)^{1 / r} \\
& \preceq \sigma_{2^{N}} \cdot 2^{N} \cdot\left(\sum_{n=0}^{N-1} 2^{r(\rho-\lambda-1) n}\right)^{1 / r} \sim \sigma_{2^{N}} \cdot 2^{(\rho-\lambda) N}
\end{aligned}
$$

where we used that $\rho-\lambda-1>0$. This gives

$$
e_{2^{N}}\left(D_{\sigma} P\right) \preceq \sigma_{2^{N}} \cdot 2^{-\lambda N} \text {. }
$$

Second summand. From (2) it follows

$$
e_{2^{N}}\left(D_{\sigma} Q\right) \leq \sigma_{2^{N}} \cdot e_{2^{N}}\left(\mathrm{id}: \ell_{p}^{2^{M}} \rightarrow \ell_{q}^{2^{M}}\right) \sim \sigma_{2^{N}} \cdot 2^{-\lambda N}(M-N)^{\lambda},
$$

and from our choice of $M, M-N \sim N^{1-\delta} \log N$, we derive

$$
e_{2^{N}}\left(D_{\sigma} Q\right) \sim \sigma_{2^{N}} \cdot\left(\frac{N^{1-\delta} \log N}{2^{N}}\right)^{\lambda} .
$$

Third summand. By Proposition 3.1 we have

$$
L_{\lambda}^{(e)}\left(D_{\sigma} R\right) \preceq \sup _{k \geq 1}(1+\log k)^{\lambda} \sigma_{2^{M}+k} \preceq \sup _{n \geq M} n^{\lambda} \sigma_{2^{n}}
$$

where we also used that $\sigma_{k} \sim \sigma_{2 k}$. Since, for $n$ and $N$ large enough, the sequence $n^{\lambda} \sigma_{2^{n}}$ is decreasing and $M \leq 2 N$, we obtain

$$
\sup _{n \geq M} n^{\lambda} \sigma_{2^{n}}=M^{\lambda} \sigma_{2^{M}} \leq(2 N)^{\lambda} \exp \left(-\alpha\left(M^{\delta}-N^{\delta}\right)\right) \sigma_{2^{N}}
$$

By the mean value theorem there exists $t \in(N, M)$ such that

$$
\frac{M^{\delta}-N^{\delta}}{M-N}=\delta t^{\delta-1} \geq \delta M^{\delta-1} \geq \delta(2 N)^{\delta-1},
$$


hence our choice of $M, M-N=\left[C N^{1-\delta} \log N\right]$ with $C=\frac{2^{1-\delta} \lambda}{\alpha \delta}$, implies

$$
\alpha\left(M^{\delta}-N^{\delta}\right) \geq \alpha \delta(2 N)^{\delta-1}(M-N)=\alpha \delta 2^{\delta-1} C \log N=\lambda \log N,
$$

and consequently

$$
\exp \left(-\alpha\left(M^{\delta}-N^{\delta}\right)\right) \leq N^{-\lambda}
$$

This gives

$$
e_{2^{N}}\left(D_{\sigma} R\right) \leq 2^{-\lambda N} L_{\lambda}^{(e)}\left(D_{\sigma} R\right) \preceq \sigma_{2^{N}} \cdot 2^{-\lambda N} .
$$

Finally, combining the relations (17)-(20), we get the upper estimate

$$
e_{3 \cdot 2^{N}}\left(D_{\sigma}\right) \preceq \sigma_{2^{N}} \cdot\left(2^{-N} N^{1-\delta} \log N\right)^{\lambda},
$$

and by a monotonicity argument the proof is finished.

\section{References}

[1] B. Carl, Entropy numbers of diagonal operators with an application to eigenvalue problems, J. Approx. Theory 32 (1981), no. 2, 135-150.

[2] B. Carl and I. Stephani, Entropy, compactness and the approximation of operators, Cambridge Tracts in Mathematics, vol. 98, Cambridge University Press, Cambridge, 1990.

[3] F. Cobos, T. Kühn, and T. Schonbek, Compact embeddings of Brézis-Wainger type, Rev. Mat. Iberoamericana, to appear.

[4] D. E. Edmunds and H. Triebel, Function spaces, entropy numbers, differential operators, Cambridge Tracts in Mathematics, vol. 120, Cambridge University Press, Cambridge, 1996.

[5] Y. Gordon, H. König, and C. Schütt, Geometric and probabilistic estimates for entropy and approximation numbers of operators, J. Approx. Theory 49 (1987), no. 3, 219-239.

[6] D. D. Haroske and H. Triebel, Wavelet bases and entropy numbers in weighted function spaces, Math. Nachr. 278 (2005), no. 1-2, 108-132.

[7] H. König, Eigenvalue distribution of compact operators, Operator Theory: Advances and Applications, vol. 16, Birkhäuser Verlag, Basel, 1986.

[8] T. Kühn, A lower estimate for entropy numbers, J. Approx. Theory 110 (2001), no. 1, $120-124$.

[9] Entropy numbers of diagonal operators of logarithmic type, Georgian Math. J. 8 (2001), no. 2, 307-318.

[10] T. Kühn, H.-G. Leopold, W. Sickel, and L. Skrzypczak, Entropy numbers of embeddings of weighted Besov spaces, Constr. Approx., to appear.

[11] _ Entropy numbers of embeddings of weighted Besov spaces II (2005), preprint.

[12] T. Kühn and T. Schonbek, Compact embeddings of Besov spaces into Orlicz and LorentzZygmund spaces, Houston J. Math., to appear.

[13] A. Pietsch, Operator ideals, Mathematische Monographien, vol. 16, VEB Deutscher Verlag der Wissenschaften, Berlin, 1978; reprinted in North-Holland, Amsterdam-New York, 1980.

[14] C. Schütt, Entropy numbers of diagonal operators between symmetric Banach spaces, J. Approx. Theory 40 (1984), no. 2, 121-128.

[15] N. Tomczak-Jaegermann, Dualité des nombres d'entropie pour des opérateurs à valeurs dans un espace de Hilbert, C. R. Acad. Sci. Paris Sér. I Math. 305 (1987), no. 7, 299-301 (French, with English summary). 